\title{
Critical State Stability and Flux Jumps' Dynamics in a Single Crystal of $\mathrm{YBa}_{2} \mathrm{Cu}_{3} \mathrm{O}_{7-\delta}$
}

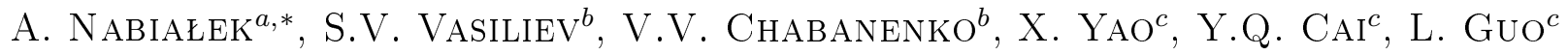 \\ AND M.V. KuZOVUI ${ }^{b}$ \\ ${ }^{a}$ Institute of Physics, PAS, al. Lotników 32/46, 02-668 Warsaw, Poland \\ ${ }^{b}$ Institute for Physics and Engineering, NASU, 72 ul. R. Luxemburg, 83114, Donetsk, Ukraine \\ ${ }^{c}$ Department of Physics, Shanghai Jiao Tong University, 800 Dong Chuan Road, Shanghai 200240, China
}

We studied the critical state stability and the dynamics of the flux jumps, caused by thermomagnetic avalanches, in a large $\left(3 \times 3 \times 1 \mathrm{~mm}^{3}\right)$ single crystal of $\mathrm{YBa}_{2} \mathrm{Cu}_{3} \mathrm{O}_{7-\delta}$ superconductor. Flux jumps were observed only at the lowest temperatures attainable in our system (at about $2.2 \mathrm{~K}$ ) and for the external magnetic field parallel to the $c$-axis of the investigated crystal. It is shown that thermomagnetic avalanches are initiated in dynamic conditions, for which the magnetic diffusivity is much smaller than the thermal diffusivity. For large single crystals of $\mathrm{YBa}_{2} \mathrm{Cu}_{3} \mathrm{O}_{7-\delta}$ immersed directly in liquid helium, the analysis of the critical state stability can be performed in isothermal approximation. However, the conditions of the critical state stability can change dramatically by improving the thermal isolation between the sample and the surrounding coolant. The dynamics of the flux jumps was analyzed in the framework of the model of magnetic diffusion. It is shown that the magnetic diffusivity changes dramatically during the process of the thermomagnetic avalanche. We also studied the influence of the external magnetic field and of the magnetic history on the duration of the flux jumps.

PACS: 74.72.-h, 74.25.Sv

\section{Introduction}

The phenomenon of thermomagnetic avalanches and the related phenomenon of giant flux jumps are problematic from the viewpoint of applications of superconductors, because they drive the superconducting sample into a normal or into a resistive state [1]. For the $\mathrm{YBa}_{2} \mathrm{Cu}_{3} \mathrm{O}_{7-\delta}$ superconductor, thermomagnetic avalanches are observed since large (of a diameter of several millimeters) single crystals or melt-textured samples are available [2-4]. Flux jumps in this material are usually observed at the temperature of about $4.2 \mathrm{~K}$. Experimental results show a strong influence of both the external magnetic sweep rate and the heat exchange conditions on flux jumping [2]. No flux jumps were observed in ceramic samples. An analysis of this phenomenon was usually performed in locally adiabatic approximation, for which the thermal diffusivity $D_{\text {th }}$ of the investigated sample is assumed to be significantly lower than the magnetic diffusivity $D_{m}\left(D_{\text {th }} \ll D_{m}\right)$. Such conditions usually occur in conventional superconductors [1]. However, the high temperature superconductors are commonly characterized by a strong flux creep phenomenon, which can be correlated with a strongly nonlinear current-voltage characteristics of these materials. It was shown by R.G. Mints [5] that, as a result of such characteristics, the conditions of the thermomagnetic avalanche development cannot be assumed as locally adiabatic. Instead, the so-called dynamic approxi-

\footnotetext{
* corresponding author; e-mail: nabia@ifpan.edu.pl
}

mation, for which $D_{m} \ll D_{\text {th }}$, should be used. An analysis of the critical state stability of the $\mathrm{YBa}_{2} \mathrm{Cu}_{3} \mathrm{O}_{7-\delta}$ superconductor in the framework of the dynamic model has not been performed so far.

The dynamics of the process of the flux jump remains until today poorly understood. However, this dynamics is important from the viewpoint of practical applications of superconductors, because large energy released during the thermomagnetic avalanche may at certain conditions cause a damage of a device which uses superconducting elements.

In the present work, we study the critical state stability and dynamics of the flux jumps in a large $\left(3 \times 3 \times 1 \mathrm{~mm}^{3}\right)$ single crystal of $\mathrm{YBa}_{2} \mathrm{Cu}_{3} \mathrm{O}_{7-\delta}$ superconductor. The critical state stability is analyzed in the framework of dynamic models. The dynamics of the flux jumps is analyzed in frames of the model of the magnetic diffusion. The influence of the magnetic history on the duration of the flux jumps is also studied.

\section{Experiment}

In our experiments, we used a $3 \times 3 \times 1 \mathrm{~mm}^{3}$ single crystal of $\mathrm{YBaCuO}_{7-\delta}$ with the $c$-axis parallel to the shortest edge of the investigated slab. $T_{\mathrm{c}}$ was about $91 \mathrm{~K}$. We observed flux jumps only for the external magnetic field parallel to the $c$-axis. Hence, all data presented here are for this sample orientation. All experiments were performed in 12 tesla superconducting magnet with variable temperature insert. At the temperature of $4.2 \mathrm{~K}$ and $2.2 \mathrm{~K}$, the sample was immersed in liquid helium. At higher temperatures, the sample was surrounded by helium gas with normal pressure. 
Our experimental setup enabled us simultaneous registration of the magnetic moment and of the changes of the magnetic flux in investigated sample. The sample was put into a vibrating sample magnetometer (VSM), which registered the magnetic moment. Additionally, a pick-up coil was wound around the investigated sample. The voltage of the coil was proportional to the time derivative of the magnetic flux in the sample. The changes of the coil voltage during flux jumps were recorded by data acquisition board (DAQ). During the following experiments, the external magnetic field was swept with the rate of about $1.5 \times 10^{-2} \mathrm{~T} / \mathrm{s}$. We also registered relaxation of the magnetic moment in constant external magnetic field $\mu_{0} H=3 \mathrm{~T}$.

\section{Results}

We observed the flux jumps at the lowest temperature attainable in our system (of about $2.2 \mathrm{~K}$ ). No flux jumps were observed neither at $4.2 \mathrm{~K}$ nor at higher temperatures. No experiments were performed in the temperature range between 2.2 and $4.2 \mathrm{~K}$. Figure 1 presents the magnetization hysteresis loop taken at $2.2 \mathrm{~K}$. One can see pronounced jumps of the magnetic moment caused by thermomagnetic avalanches. Dynamics of the flux jumps marked in Fig. 1 as A, B, C, D and E is shown in Fig. 2. This figure shows a time dependence of the voltage of the pick-up coil (wound around the investigated sample) during the successive flux jumps. The maximal voltage per one coil during the flux jumps ranges form about $40 \mathrm{mV}$ (Fig. 2, curve D) to about $1.5 \mathrm{~V}$ (Fig. 2, curve A). The structure of the flux jumps is complex. They consist of several peaks in voltage. The widths of the peaks as well as the distance between the peaks is of an order of 10 $20 \mu \mathrm{s}$. The peaks are followed by a long "tail" of relatively slowly decreasing voltage.

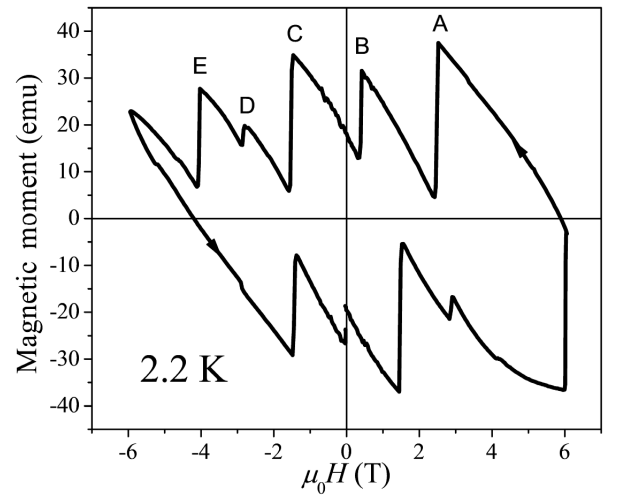

Fig. 1. The magnetization hysteresis loop of a $3 \times 3 \times$ $1 \mathrm{~mm}^{3} \mathrm{YBa}_{2} \mathrm{Cu}_{3} \mathrm{O}_{7-\delta}$ crystal taken at $2.2 \mathrm{~K}$. Dynamics of the flux jumps denoted as A, B, C, D and E is shown in Fig. 2.

\section{The stability of the critical state}

We analyzed models of the critical state stability for an infinitely large superconducting slab with the width

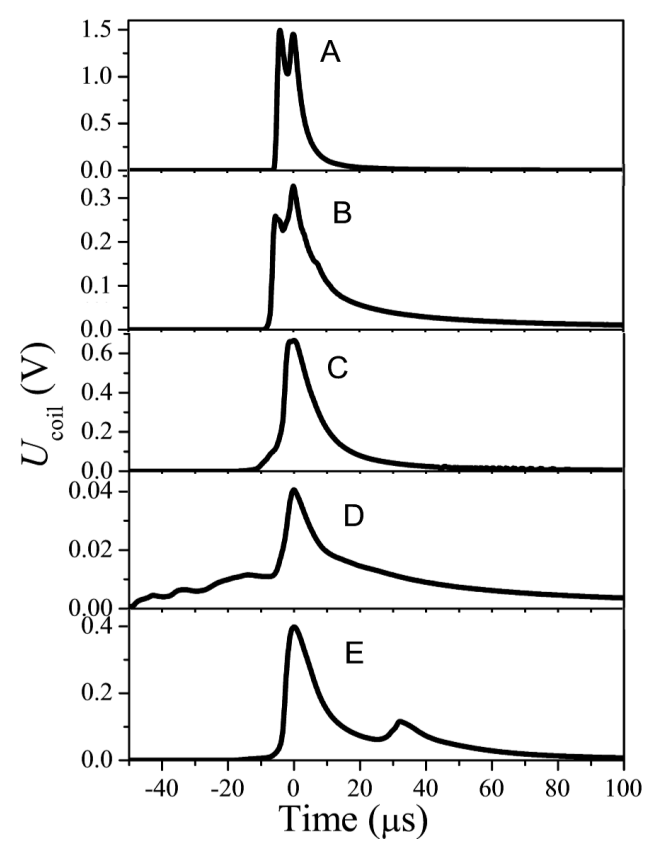

Fig. 2. Time dependence of the voltage per one turn of the pick-up coil wound around the $\mathrm{YBa}_{2} \mathrm{Cu}_{3} \mathrm{O}_{7-\delta}$ crystal during the successive flux jumps, denoted in Fig. 1 as $\mathrm{A}, \mathrm{B}, \mathrm{C}, \mathrm{D}$ and $\mathrm{E}$.

$2 d$ (in our case $d=1.5 \mathrm{~mm}$ ). We calculated the critical dimension of the sample $d_{\text {crit }}$ it means the minimal dimension of the superconducting sample for which, at given temperature, we may expect the flux jumps to occur. This critical dimension can by correlated with the field at which the first flux jump, after cooling the sample in zero magnetic field, occur $-H_{\mathrm{fj} 1}=j_{\mathrm{c}} d_{\text {crit }}$. For several limiting cases, critical dimension of the superconducting sample (or $H_{\mathrm{fj} 1}$ ) can be expresses by analytical formulas.

a) In locally adiabatic conditions [1]

$$
d_{\mathrm{crit}}^{\mathrm{ad}}=\sqrt{\frac{2 c_{V}}{\mu_{0} j_{\mathrm{c}}\left|\frac{\partial j_{\mathrm{c}}}{\partial T}\right|}},
$$

where $c_{V}$ is the specific heat, $\mu_{0}$ - magnetic permeability of vacuum and $j_{\mathrm{c}}-$ critical current density. This formula can be applied if $D_{\text {th }} \ll D_{m} . \quad D_{\text {th }}=\frac{\kappa}{c_{V}}$ and $\kappa$ is the thermal conductivity. $D_{m}=\frac{\rho}{\mu_{0}}$ and $\rho$ is the resistivity.

b) Dynamic conditions

Such conditions occur, if $D_{\text {th }} \gg D_{m}$. In this case, the stability of the critical state depends on the relation between the sample dimensions, thermal conductivity of the sample and the boundary thermal conductivity $h$. The critical state stability criterion can be expressed by analytical formulas in two limiting cases:

If $B i=\frac{d h}{\kappa} \ll 1$ ( $B i$ is so-called Biot number), we can use the approximation of a weakly cooled sample. In this approximation, we can assume the thermal perturbations to develop uniformly in the whole sample volume [5]. We can assume the current-voltage characteristic of the superconducting sample in a form 


$$
j(E)=j_{\mathrm{c}}+\frac{j_{\mathrm{c}}}{n} \ln \left(\frac{E}{E_{0}}\right),
$$

where $E_{0}$ is the voltage criterion at which the critical current density $j_{\mathrm{c}}$ is defined, and $n$ is a dimensionless parameter. One usually assumes $E_{0}=10^{-4} \mathrm{~V} / \mathrm{m}$. In this case $n \gg 1$. The critical dimension of the sample can be expressed by the formula [5]:

$$
d_{\mathrm{crit}}^{\mathrm{wc}}=\sqrt{\frac{2 h}{\mu_{0} n\left|\frac{\partial j_{\mathrm{c}}}{\partial T}\right|\left(\frac{\partial H}{\partial t}\right)}},
$$

where $\left(\frac{\partial H}{\partial t}\right)$ is the external magnetic field sweep rate.

If $B i=\frac{d h}{\kappa} \gg 1$, we can analyze the stability of the critical state in isothermal approximation. In this case

$$
d_{\mathrm{crit}}^{\text {is }}=\sqrt[3]{\frac{2 \kappa}{\gamma \mu_{0} n\left|\frac{\partial j_{\mathrm{c}}}{\partial T}\right|\left(\frac{\partial H}{\partial t}\right)}},
$$

where $\gamma \approx 2.48[6]$.

We calculated temperature dependence of the critical current density $j_{\mathrm{c}}(T)$ as proportional to the widths of the magnetization hysteresis loops. In the external magnetic field $\mu_{0} H=3 \mathrm{~T}$, and in the range of temperatures from $2 \mathrm{~K}$ to $20 \mathrm{~K}$, this dependence was fitted according to the experimental formula $j_{c}(T)=j_{c 0} \exp \left(c_{1} T+c_{2} T^{2}\right)$ with $j_{\mathrm{c} 0} \approx 1.03 \times 10^{10} \mathrm{~A} / \mathrm{m}^{2}, c_{1} \approx-0.106 \mathrm{~K}^{-1}$ and $c_{2} \approx 1.19 \times 10^{-3} \mathrm{~K}^{-2}$. Temperature and magnetic field dependence of the specific heat $c_{V}$ was taken from [7] (see data for sample T1 in Ref. [7]).

There is a large difference between the experimental data of the thermal conductivity $\kappa$ reported by different authors. Studies in the low temperature regime [8] reveal the in plane thermal conductivity at $T=1 \mathrm{~K}$ of about $10^{-1} \mathrm{~W} / \mathrm{Km}$. At the temperature of $10 \mathrm{~K}$, the reported in-plane thermal conductivity ranges form $1.6 \mathrm{~W} / \mathrm{Km}$ [9] to about $6 \mathrm{~W} / \mathrm{Km}[10]$. In order to fit these experimental data in the temperature range form $1 \mathrm{~K}$ to $20 \mathrm{~K}$, we used a power function $\kappa(T)=10^{-1} T^{x}$, where $x \approx 1.2$ for the data taken from [9] and $x \approx 1.7$ for the data taken from [10]. In our further analysis, we will use the average value $x=1.5$.

The boundary thermal conductivity $h$ is very difficult to determine experimentally, and it depends on many factors (e.g. on sample roughness). If the boundary conductivity is governed by phonon processes, $h(T) \sim$ $T^{3}$ [11]. For a sample immersed directly in helium bath, $h \approx 10^{4} \mathrm{~W} / \mathrm{m}^{2} \mathrm{~K}$ [1]. However, after improving the thermal isolation, this parameter may decrease by several orders of magnitude.

It is impossible to measure directly the current-voltage characteristic of our sample. In order to reach the critical current density of an order of $10^{10} \mathrm{~A} / \mathrm{m}^{2}$, we should supply our sample with the current of an order of $10^{4} \mathrm{~A}$. Hence in order to determine the current-voltage characteristic of the investigated sample, we applied an indirect method. We measured relaxation of the magnetic moment in the external magnetic field $\mu_{0} H=3 \mathrm{~T}$. The magnetic moment, whose absolute value decreases in time, is proportional to the density of the current screening the sample volume. On the other hand, time derivative of the sample magnetization is proportional to the electric field induced at the sample surface. Treating the time as a parameter, we can obtain the current-voltage characteristic. This characteristic was next fitted according to Eq. (2), in order to determine the $n$-parameter, which stands in Eqs. (3) and (4). In the external magnetic field $\mu_{0} H=3 \mathrm{~T}$, the $n$-parameter decreased with increasing temperature from about 70 at $2.2 \mathrm{~K}$ to about 30 at $20 \mathrm{~K}$. We fitted the $n(T)$ dependence according to experimental formula $n(T)=97.1 \times T^{-0.52}$.

The relation between thermal and magnetic diffusivity is usually described using the parameter $\tau=D_{\text {th }} / D_{m}$. $\tau \ll 1$ for locally adiabatic conditions, and $\tau \gg 1$ for dynamic conditions. For the current-voltage characteristic described by Eq. (2), $\rho=\frac{n E}{j_{\mathrm{c}}}$. The electric field at the surface of the slab $E=\mu_{0} d\left(\frac{\partial H}{\partial t}\right)$. Hence, $\tau=\frac{\kappa j_{\mathrm{c}}}{c_{V} n d\left(\frac{\partial H}{\partial t}\right)}$. Taking the experimental data, we can find that the $\tau$ parameter decreases from about $10^{4}$ at $2 \mathrm{~K}$ to about $3 \times 10^{2}$ at $20 \mathrm{~K}$. The magnetic diffusivity at the initial stage of the thermomagnetic avalanche $D_{m} \sim 10^{-7} \mathrm{~m}^{2} / \mathrm{s}$. In this range of temperatures $\tau \gg 1$ and we expect the thermomagnetic avalanche to develop in dynamic conditions.

If we assume for the sample immersed in liquid helium $h(4.2 \mathrm{~K}) \approx 10^{4} \mathrm{~W} / \mathrm{m}^{2} \mathrm{~K}$, we obtain $\operatorname{Bi}(4.2 \mathrm{~K}) \approx 17>1$. Hence around $4.2 \mathrm{~K}$, the stability of the critical state should be better described by the isothermal approximation. However, if $h$ decreases by at least two orders of magnitude, $B i<1$ in the whole range $2-20 \mathrm{~K}$, and we expect the stability of the critical state to be better described be the approximation of the weakly cooled sample.

We used Eqs. (1), (3) and (4) in order to calculate temperature dependence of the critical dimension $d_{\text {crit }}(T)$ in the range $0-20 \mathrm{~K}$ (see Fig. 3). Dashed line in Fig. 3 corresponds to the half-width of the investigated sample $d$. Flux jumps may occur only if $d>d_{\text {crit }}$. Hence, in the case of our sample immersed directly in liquid helium (isothermal conditions), we can expect flux jumps only for the temperatures lower than $4 \mathrm{~K}$. This is in good agreement with the experimental results, where we observed flux jumps at $2.2 \mathrm{~K}$ and no jumps at $4.2 \mathrm{~K}$. For the approximation of the weakly cooled sample (Eq. (3)), we assumed $h(T)=c_{3} T^{3}$. We present here three curves calculated for the approximation of the weakly cooled sample (see dotted lines in Fig. 3). The curves were calculated assuming $c_{3}=1,0.1$ or $0.01 \mathrm{~W} / \mathrm{m}^{2} \mathrm{~K}^{4}$, respectively. At the temperature of $4.2 \mathrm{~K}$, these three coefficients correspond to the boundary thermal conductivity equal to 74 , 7.4 or $0.74 \mathrm{~W} / \mathrm{m}^{2} \mathrm{~K}$, respectively, which is approximately two, three or four orders of magnitude lower than for the sample immersed in liquid helium. One can see that by improving the thermal isolation, it means after lowering the $h$-parameter, temperature range of the flux jump occurrence increases significantly. The predictions of the 
locally adiabatic approximation are also shown in Fig. 3. However as we discussed above, they are not realistic for the conditions of our experiment.

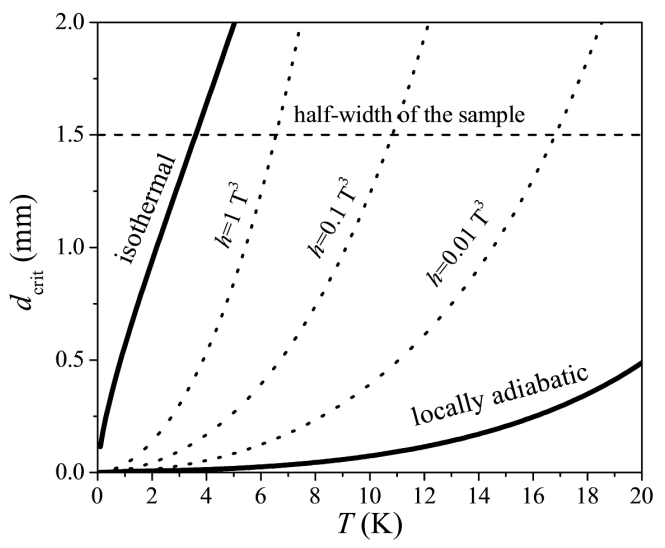

Fig. 3. Critical, from the viewpoint of the occurrence of thermomagnetic avalanches, dimension $d_{\text {crit }}$ of the $\mathrm{YBa}_{2} \mathrm{Cu}_{3} \mathrm{O}_{7-\delta}$ slab as a function of temperature calculated in the framework of different approximations: isothermal, locally adiabatic and of the weakly cooled sample. In the approximation of the weakly cooled sample (dotted lines) the boundary thermal conductivity, $h(T)=c_{3} T^{3}$ and $c_{3}=1,0.1$ or $0.01 \mathrm{~W} / \mathrm{m}^{2} \mathrm{~K}^{4}$, respectively. The half-width of the sample studied in experiment, $d=1.5 \mathrm{~mm}$, is marked by a dashed line. Thermomagnetic avalanches occur if $d>d_{\text {crit }}$.

Our analysis of the critical state stability was performed in the framework of models developed for infinite slab sample. Hence, for our sample such analysis can be treated as a rough approximation. We studied the critical state stability only in one crystalline sample of $\mathrm{YBa}_{2} \mathrm{Cu}_{3} \mathrm{O}_{7-\delta}$. Other experiments of the flux jumps in single crystals or melt-textured samples of $\mathrm{YBa}_{2} \mathrm{Cu}_{3} \mathrm{O}_{7-\delta}$ superconductor [2-4] reveal flux jumps also at the temperature of $4.2 \mathrm{~K}$ as well as at higher temperatures (up to about $7.5 \mathrm{~K}$ in Ref. [4]). Unfortunately, in the case of these experiments, it is impossible to perform an analysis of the critical state stability, because of the lack of some important data, e.g. the current-voltage characteristics of the investigated samples. The fact that flux jumps in Ref. [4] were also observed at the temperature of about $7.5 \mathrm{~K}$ is probably connected with different cooling conditions - the experiment was performed in SQUID magnetometer. We observed a strong influence of the cooling condition on the range of the flux jumps occurrence in a single crystal of $\mathrm{La}_{1.85} \mathrm{Sr}_{0.15} \mathrm{CuO}_{4}$ superconductor [6].

\section{Dynamics of the flux jumps}

Let us consider a slab with diameter $2 d$ and the initial distribution of the induction of the local magnetic field $B(x)$. We assume $x=0$ in the center of the slab and $x= \pm d$ at the sample walls. If the dynamics of the magnetic flux in the sample is governed by the process of magnetic diffusion, we can express the voltage per one coil wound around the investigated sample by the following formula:

$$
\begin{aligned}
& U_{\text {coil }}(t)=\frac{4 D_{m} L}{d} \\
& \quad \times \sum_{k=0}^{\infty} A_{k} \exp \left(-\frac{D_{m}(1+2 k)^{2} \pi^{2} t}{4 d^{2}}\right),
\end{aligned}
$$

where $L$ is the length of the slab and $A_{k}=$ $\frac{1}{d} \int_{-d}^{d} B(x) \cos \left(\frac{(2 k+1) \pi x}{2 d}\right) \mathrm{d} x$. In further analysis, we assume the dynamics of the flux jumps to be governed by the first harmonic (with $k=0$ ) and $U_{\text {coil }}(t) \sim$ $\exp \left(-\frac{D_{m} \pi^{2} t}{4 d^{2}}\right)$. According to the model of the magnetic diffusion, the voltage of the coil decreases in time. We applied this model to determine the magnetic diffusivity at the final stage of the flux jump (for decreasing $U_{\text {coil }}(t)$ ). We assumed $t=0$ for the maximum of the peak in voltage. The results of our analysis are shown in Fig. 4. One can see that at the final stage of the flux jump, magnetic diffusivity decreases from about $10^{-1} \mathrm{~m}^{2} / \mathrm{s}$ (time of an order of $10^{-5} \mathrm{~s}$ ) to about $10^{-3} \mathrm{~m}^{2} / \mathrm{s}$ (time of an order of $10^{-3} \mathrm{~s}$ ). It is worth to notice that the in-plane normal state resistivity $\rho_{\mathrm{n}}^{\mathrm{ab}}\left(T=T_{\mathrm{c}}\right) \approx 3 \times 10^{-6} \Omega \mathrm{m}[12]$. Hence, $D_{m}\left(T=T_{\mathrm{c}}\right) \approx 2.4 \mathrm{~m}^{2} / \mathrm{s}$ is approximately one order of magnitude higher than the maximal magnetic diffusivity observed in our experiments.

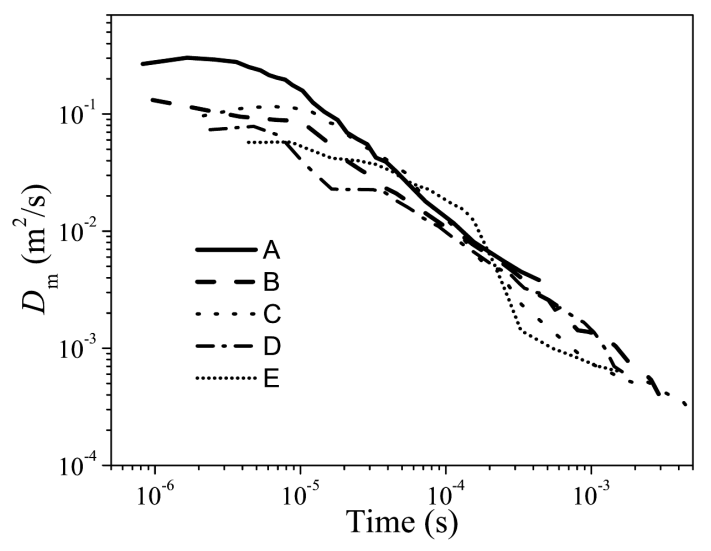

Fig. 4. Time dependence of the magnetic diffusivity $D_{m}$ at the final stage of the flux jump. The A, B, C, $\mathrm{D}$ and $\mathrm{E}$ curves were calculated according to time dependence of the A, B, C, D and E voltages presented in Fig. 2.

We can understand the experimentally observed dynamics of the flux jumps as follows. At the beginning of the flux jump process, the sample is in the flux creep regime characterized by a very small (of an order of $10^{-7} \mathrm{~m}^{2} / \mathrm{s}$ ) magnetic diffusivity, and the flux jump is initiated in dynamic conditions. When the temperature of the sample, as a result of the thermomagnetic avalanche development, increases, the magnetic diffusivity increases by six orders of magnitude to the value of an order of $10^{-1} \mathrm{~m}^{2} / \mathrm{s}$. This value of the magnetic diffusivity is lower than that typical for the normal state. It means that maximal temperature of the sample dur- 
ing the thermomagnetic avalanche is lower than $T_{\mathrm{c}}$. The voltage induced by the flux jump increases from about $10^{-7} \mathrm{~V}$ (the voltage induced by the external magnetic field sweep) to about $1 \mathrm{~V}$ in a time of an order of $10^{-5} \mathrm{~s}$. Such a rapid increase in voltage may be dangerous in some technical application of the superconductors. At the final stage of the flux jump, the magnetic diffusivity decreases to about $10^{-4}-10^{-3} \mathrm{~m}^{2} / \mathrm{s}$. This decrease can be connected with a decrease of the sample temperature to the temperature of the surrounding bath. The diffusivity of an order of $10^{-4}-10^{-3} \mathrm{~m}^{2} / \mathrm{s}$ is typical for thermal processes in the temperature range $2-20 \mathrm{~K}$.

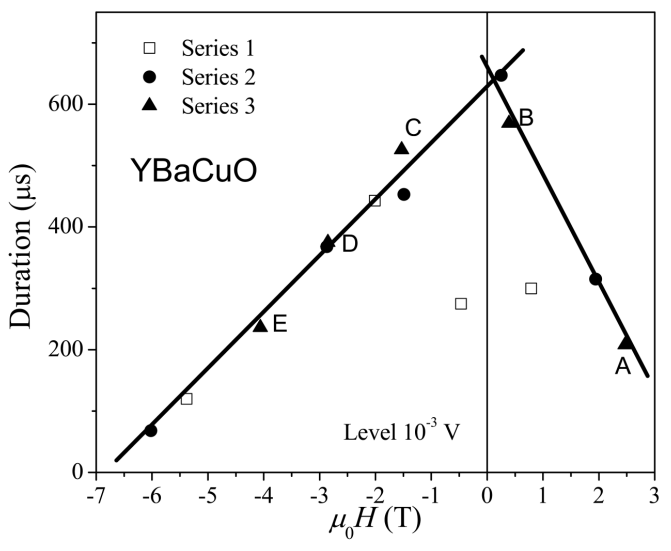

Fig. 5. Duration of the successive flux jumps determined at the voltage level $10^{-3} \mathrm{~V}$ as a function of the external magnetic field. Figure presents three series of measurements for the upper branch of the magnetization hysteresis loop. Letters A, B, C, D and E of the series 3 correspond to the flux jumps presented in Figs. 1 and 2 .

We also study the influence of the magnetic history and of the external magnetic field on the duration of the successive flux jumps. Duration of the flux jumps depends on the voltage level at which we determine the begin and the end of the flux jump. This duration was of an order of $10^{-5}-10^{-4} \mathrm{~s}$ at the voltage level of $10^{-2} \mathrm{~V}$ and of an order of $10^{-4}-10^{-3} \mathrm{~s}$ at the voltage level of $10^{-3} \mathrm{~V}$. We repeated the experiments several times, and we observed a large scatter of the experimental results. This scatter is probably connected with the complex structure of the observed flux jumps. However, most of the series of experiments suggested an increase of the duration of the flux jumps in zero magnetic field. Such increase can be understood, if we correlate the magnetic diffusivity with the flux flow resistivity $\rho_{\mathrm{ff}}=\rho_{\mathrm{n}}\left(\frac{H}{H_{\mathrm{c} 2}}\right)$, where $\rho_{\mathrm{n}}$ is the normal state resistivity and $H_{\mathrm{c} 2}$ is the upper critical field, because with a decrease of the magnetic diffusivity (in lower magnetic field), we expect an increase of the duration of the flux jumps. Figure 5 shows duration of the flux jumps measured at the voltage level $10^{-3} \mathrm{~V}$ as a function of the external magnetic field. All series presented in Fig. 5 are for the upper branch of the mag- netization hysteresis loop. Letters A, B, C, D and E in series 3 correspond to the flux jumps presented in Fig. 1 and Fig. 2.

At the end, we want to emphasize that, because of a complex structure of the thermomagnetic avalanche, a simple model of the magnetic diffusion is insufficient to describe all features of the dynamics of this process.

\section{Conclusions}

For the crystal of $\mathrm{YBa}_{2} \mathrm{Cu}_{3} \mathrm{O}_{7-\delta}$ studied in our experiments, thermomagnetic avalanches were initiated in dynamic conditions, for which the magnetic diffusivity in the flux creep regime (of an order of $10^{-7} \mathrm{~m}^{2} / \mathrm{s}$ ) was much smaller than the thermal diffusivity (of an order of $10^{-3} \mathrm{~m}^{2} / \mathrm{s}$ ). In the case of our relatively large (with a diameter of about $3 \mathrm{~mm}$ ) crystal immersed in helium bath, in order to calculate the critical state stability criterion, we can use the isothermal approximation. In such conditions flux jumps occur only below $4.2 \mathrm{~K}$. However, temperature range of the flux jumps occurrence can increase significantly after improving the thermal isolation of the sample. The voltage induced by the flux jump increases from about $10^{-7} \mathrm{~V}$ to about $1 \mathrm{~V}$ in a time of an order of $10^{-5} \mathrm{~s}$.

\section{Acknowledgments}

This work was partly supported by Polish National Science Centre under research project for years 20112012 (grant N N202 1663 40).

\section{References}

[1] R.G. Mints, A.L. Rakhmanov, Rev. Mod. Phys. 53, 551 (1981).

[2] M. Guillot, M. Potel, P. Gougeon, H. Noel, J.C. Levet, G. Chouteau, J.L. Tholence, Phys. Lett. A 127, 363 (1988).

[3] K. Watanabe, N. Kobayashi, S. Awaji, G. Kido, S. Nimori, K. Kimura, K. Sawano, Y. Muto, Jpn. J. Appl. Phys., Part 2 30, L1638 (1991).

[4] K.H. Müller, C. Andrikidis, Phys. Rev. B 491294 (1994).

[5] R.G. Mints, Phys. Rev. B 53, 12311 (1996).

[6] A. Nabiałek, A. Wiśniewski, V.V. Chabanenko, S.V. Vasiliev, T.V. Tsvetkov, F. Pérez-Rodríguez, Supercond. Sci. Technol. 25, 035005 (2011).

[7] K.A. Moler, D.L. Sisson, J.S. Urbach, M.R. Beasley, A. Kapitulnik, D.J. Baar, R. Liang, W.N. Hardy, Phys. Rev. B 55, 3954 (1997).

[8] G. Sparn, M. Baenitz, S. Horn, F. Steglich, W. Assmus, T. Wolf, A. Kapitulnik, Z.X. Zhao, Physica $C$ 162-164, 508 (1989).

[9] V. Gusakov, A. Jezowski, S. Barilo, N. Kalanda, A. Saiko, Physica C 341-348, 1867 (2000).

[10] H. Fujishiro, M. Ikebe, T. Naito, K. Noto, S. Kohayashi, S. Yoshizawa, Jpn. J. Appl. Phys. 33, 4965 (1994).

[11] E.T. Swarz, R.O. Pohl, Rev. Mod. Phys. 61, 605 (1989).

[12] H. Fujishiro, H. Teshima, M. Ikebe, K. Noto, Physica C 392-396, 171 (2003). 